\title{
Hidden-variable theorems for real experiments
}

\author{
Christoph Simon ${ }^{1,2}$, Časlav Brukner ${ }^{1}$, and Anton Zeilinger ${ }^{1}$ \\ ${ }^{1}$ Institut für Experimentalphysik, Universität Wien, Boltzmanngasse 5, A-1090 Wien, Austria \\ 2 Centre for Quantum Computation, Clarendon Laboratory, University of Oxford, Parks Road, Oxford OX1 3PU, United \\ Kingdom
}

(October 25, 2018)

It has recently been questioned whether the KochenSpecker theorem is relevant to real experiments, which by necessity only have finite precision. We give an affirmative answer to this question by showing how to derive hiddenvariable theorems that apply to real experiments, so that non-contextual hidden variables can indeed be experimentally disproved. The essential point is that for the derivation of hidden-variable theorems one does not have to know which observables are really measured by the apparatus. Predictions can be derived for observables that are defined in an entirely operational way.

In general quantum mechanics only makes probabilistic predictions for individual events. The question whether one can go beyond quantum mechanics in this respect has been a subject of debate and research since the early days of the theory. There are famous theorems placing restrictions on possible hidden-variable theories reproducing the results of quantum mechanics. Bell's theorem [1] excludes local hidden-variables. The Kochen-Specker [2] theorem excludes non-contextual hidden variables. In local hidden-variable theories the predetermined results for a given measurement are independent of which measurements are performed at space-like separation. In non-contextual hidden-variable theories the pre-determined results are independent of any measurements that are performed jointly.

The present work is concerned with the derivation of hidden-variable theorems that apply to real experiments. It was motivated by the thought-provoking work of Meyer [3], who claimed that the Kochen-Specker theorem was "nullified" for real experiments because of the unavoidably finite measurement precision, i.e. because the observer does not have full control over his experimental setup. However, our approach is very general and applies in a much broader context. It allows one to deal with all possible experimental imperfections, and it is not restricted to the specific class of non-contextual hidden variables. In particular, theorems on local hidden variables can be derived in the same way.

Let us begin our discussion with some remarks on experimental tests of hidden variables in general. Hiddenvariable theorems provide us with predictions made by certain classes of hidden-variable theories which can be tested experimentally and which differ from the quantum mechanical predictions. For local hidden variables,
Bell's inequalities can be derived without making any reference to quantum mechanics. Then one can check whether they are fulfilled by the quantum mechanical predictions, and by experiment. For non-contextual hidden variables, one can use the Kochen-Specker argument to derive specific quantitative predictions for the results of experiments, which can then be compared to quantum mechanics and to nature. For experimental proposals and Kochen-Specker type experiments that were performed in this spirit see 何.

It is sometimes argued that the Kochen-Specker theorem just makes a conceptual point about the formal structure of quantum mechanics and that actually performing experimental tests for non-contextual hidden variables is unnecessary, since quantum mechanics is a very well tested theory. Irrespective of this discussion, we feel that the question whether such tests could be performed in principle is interesting because it concerns the falsifiability of a rather simple and fundamental concept.

In the derivation of hidden-variable theorems frequently no direct reference to experiments is made, or the experimental situation is treated in an idealized way. For example, in the original derivation of the KochenSpecker theorem it was just shown that non-contextual hidden variables are incompatible with quantum mechanics, without making the experimental predictions of noncontextual hidden variables explicit, while in the original derivation of Bell's theorem perfect correlations and perfect detection efficiency were assumed.

If one wants to consider an experimental test for a certain class of hidden-variable theories, one has to give up these idealized assumptions and derive hidden-variable theorems that apply to the true experimental situation. For local hidden variables, the case of non-perfect correlations and detection efficiency was analyzed soon after Bell's original derivation, starting with the work of Clauser and Horne [5]. The same kind of analysis is possible for non-contextual hidden variables.

However, there is one more idealization made in the usual derivations of hidden-variable theorems which, to our knowledge, has never been discussed in an explicit way, namely the accuracy with which the experimental setup can be maintained (e.g. how well the Stern-Gerlach magnets in a spin measurement can be aligned and kept stable). This question has become particularly important for the Kochen-Specker theorem in view of recent claims 
by Meyer [3] that this theorem is no longer applicable when the measurements have only finite precision.

In its original form, the Kochen-Specker theorem states that is is impossible to assign values to observables corresponding to all directions on the sphere subject to a constraint for triads of orthogonal directions. The observables under consideration are the squares of the spin components of a spin-1 particle along the respective direction, and the constraint is given by the total spin.

Meyer's claim was based on the fact that it is possible to assign values compatible with the constraint to all rational directions on the sphere, which constitute a dense subset of all directions [6]. He argued that, since measurements with finite precision cannot discriminate a dense subset from its closure, this implies that noncontextual hidden variables cannot be excluded by any real Kochen-Specker type experiment. However, he did not construct an explicit non-contextual hidden-variable model for real experiments with finite precision [10].

At first sight, it does not seem possible to refute Meyer's claim on the basis of existing theorems, for the following reason. An essential feature of all hiddenvariable theorems known to us is that some observables have to appear in different experimental contexts: an observable $A$ has to be measured together with another observable $B$ on a sample of systems, and also together with a third observable $C$ on another sample of systems from the same source, where both $B$ and $C$ commute with $A$, but they do not commute with each other and thus cannot be measured jointly. Note that even for tests of non-contextuality it is not necessary to perform several incompatible measurements on the same system, which would of course be impossible [7].

For example, in the original Kochen-Specker situation, one can only arrive at a contradiction by considering several triads of directions that have at least some directions in common. For Kochen-Specker experiments this implies that, at least for some directions, the observables corresponding to these directions have to appear in different triads. When the finite precision of real experiments is taken into account, it seems impossible to ascertain that the same observable is really measured more than once in different experimental contexts. Thus the usual derivations of hidden-variable theorems seem to run into problems.

Does this mean that it is impossible in principle to rule out non-contextuality experimentally? Could even the experimental results on local hidden variables be in danger for the same reasons? We are going to show that the answer is no. There are predictions of non-contextual hidden variables, which can be derived within a framework that is sufficiently general to apply to real experiments, and which can therefore also be tested by real experiments with sufficiently high, but finite, precision.

In order to achieve this, it is important to realize that predictions for classes of hidden-variable theories can be derived without reference to quantum mechanics. In such an approach, the observables playing a role in the hiddenvariable predictions are not defined via the quantummechanical formalism, but in an operational way.

For concreteness, imagine that an observer wants to perform a measurement of the spin square of a spin-1 particle along a certain direction $\vec{n}$. There will be an experimental procedure for trying to do this as accurately as possible. We will refer to this procedure by saying that he sets the "control switch" of his apparatus to the position $\vec{n}$. In all experiments that we will discuss only a finite number of different switch positions is required. By definition different switch positions are clearly distinguishable for the observer, and the switch position is all he knows about. Therefore, in an operational sense the measured physical observable is entirely defined by the switch position. From the above definition it is clear that the same switch position can be chosen again and again in the course of an experiment (while of course the system measured will always be different, cf. [7]).

In general one has to allow for the possibility that the switch position $\vec{n}$ does not uniquely determine the physical state of the measuring apparatus, i.e. there may be degrees of freedom of the apparatus over which the observer does not have full control but which may influence the result of any given measurement. In the context of deriving hidden-variable theorems, this possibility can be accomodated in a very simple and general way. Following the philosophy of deterministic hidden variable theories [8], there must also be some (in general hidden, i.e. unknown) variables determining the behaviour of the apparatus, and one has to assume that the result of any measurement will be determined by the hidden variables of the system and by those of the apparatus together.

Notice that in such an approach as described in the two preceding paragraphs it does not matter which observable is "really" measured by the apparatus and to what precision. One just derives general predictions for the behaviour of system and apparatus together, provided that certain switch positions are chosen. These predictions only depend on the properties of the class of hidden-variable theories considered. The question of the correct quantum-mechanical description of the non-ideal measurement considered arises only when, as a next step, one wants to obtain the quantum-mechanical predictions for the given situation.

Following the method described above, we will now show how non-contextual hidden variables can be tested and thus potentially excluded by real experiments. We will consider the context of the original Kochen-Specker argument, i.e. exactly the case considered by Meyer.

In the original Kochen-Specker situation one considers a spin-1 particle. In the ideal case of perfect precision, the relevant quantum-mechanical observables are the squares of the spin components, denoted by $\hat{S}_{\vec{n}}^{2}$ for arbitrary directions $\vec{n}$. For a spin-1 particle one has 


$$
\hat{S}_{\vec{n}_{1}}^{2}+\hat{S}_{\vec{n}_{2}}^{2}+\hat{S}_{\vec{n}_{3}}^{2}=2
$$

for every triad of orthogonal directions $\left\{\vec{n}_{1}, \vec{n}_{2}, \vec{n}_{3}\right\}$. As the possible results for every $\hat{S}_{\vec{n}_{i}}^{2}$ are 0 or 1 , this implies that in the ideal case for every measurement of three orthogonal spin squares two of the results will be equal to one, and one of them will be equal to zero.

Let us emphasize that in our approach, in the derivation of the hidden-variable predictions, the observables are defined operationally by the switch positions, i.e. by the best effort and knowledge of the experimenter, and cannot be identified with the quantum-mechanical observables. Of course, for a specific experiment, there should be some approximate correspondence in order to ensure that the quantum-mechanical predictions will be sufficiently close to the ideal case so that they are still in conflict with the relevant hidden-variable predictions. In the following the symbol $S_{\vec{n}}^{2}$ (without the hat) will denote the operational observable defined by the switch position $\vec{n}$, and the term direction will be used as a synonym for switch position.

In a deterministic hidden variable theory (cf. [8]) one assumes that for every individual particle the result of the measurement of any observable $S_{\vec{n}}^{2}$ is predetermined by hidden variables. In non-contextual hidden variable theories it is furthermore assumed that this predetermined result does not depend on the "context" of the measurement, i.e. on which other observables are measured together with $S_{\vec{n}}^{2}$, but only on the switch position $\vec{n}$ and the hidden variables [9].

In general the result may depend both on the hidden variables of the system and of the apparatus. Let us denote the hidden variables of the system by $\lambda$ and those of the apparatus by $\mu$. As explained above, the philosophy of non-contextual hidden variables implies the existence of a function $S_{\vec{n}}^{2}(\lambda, \mu)$ taking values 0 and 1 which describes the result of a measurement with switch position $\vec{n}$ on a system characterized by $\lambda$ with an apparatus characterized by $\mu$. For fixed $\lambda$ and $\mu$ this function therefore assigns a value 0 or 1 to the switch position $\vec{n}[10]$.

A Kochen-Specker experiment can now be performed by testing the validity of Eq. (11) (without hats) for a judiciously chosen set of triads of directions. Therefore the apparatus is required to have three switches where the three directions of a given triad can be chosen. Because the switch positions do not correspond to the ideal quantum mechanical observables the sum of the three results will not always be equal to 2 . Nevertheless a contradiction between non-contextuality and quantum mechanics can be obtained in the following way.

From the Kochen-Specker theorem it follows that there are finite sets of triads for which no value assignment consistent with Eq. (11) (again without the hats) is possible [2.11. Let us choose such a Kochen-Specker set of triads

$$
\left\{\left\{\vec{n}_{1}, \vec{n}_{2}, \vec{n}_{3}\right\},\left\{\vec{n}_{1}, \vec{n}_{4}, \vec{n}_{5}\right\}, \ldots,\right\} .
$$

Let us emphasize that at least some of the switch positions $\vec{n}_{i}$ have to appear in several of the triads; clearly otherwise there could be no inconsistency. Let us denote the number of triads in the Kochen-Specker set (2) by $N$. The set is constructed in such a way that for any fixed values of $\lambda$ and $\mu$ the equation

$$
S_{\vec{n}_{i}}^{2}(\lambda, \mu)+S_{\vec{n}_{j}}^{2}(\lambda, \mu)+S_{\vec{n}_{k}}^{2}(\lambda, \mu)=2
$$

must be violated for at least one out of the $N$ triads $\left\{\vec{n}_{i}, \vec{n}_{j}, \vec{n}_{k}\right\}$.

Suppose that one can establish experimentally that for all triads in the Kochen-Specker set the sum of the results is equal to 2 in a fraction greater than $1-\epsilon$ of all cases. For the hidden variables this implies that Eq. (3) must hold for a fraction $1-\epsilon$ of all $(\lambda, \mu)$, for all triads in the set. But for sufficiently small $\epsilon$ this implies that there would have to be pairs $(\lambda, \mu)$, for which Eq. (3) holds for all triads in the set, which is impossible according to the Kochen-Specker argument.

To determine the required value for $\epsilon$, it is convenient to use a set-theoretic language. Let us denote the set of all pairs of hidden variables $(\lambda, \mu)$ by $\Lambda$. Furthermore let us denote the subset of hidden variables for which the sum of spin squares is equal to 2 for the $k$-th triad by $\Lambda_{k}$. The value of $\epsilon$ has to be sufficiently small such that the intersection of all the $\Lambda_{k}$ cannot be empty. If we define the measure (i.e. the size in terms of probability) of $\Lambda$ to be 1 , then according to our assumptions all the $\Lambda_{k}$ have measures larger than $1-\epsilon$, which implies that the measure of the intersection of all the $\Lambda_{k}$ is larger than $1-N \epsilon$. This follows from the fact that the complement of each $\Lambda_{k}$ is smaller than $\epsilon$, such that the size of the union of these complements, which is the complement of the intersection of all the $\Lambda_{k}$, cannot be larger than $N \epsilon$.

Thus non-contextuality is experimentally disproved as soon as $\epsilon$ is smaller than $1 / N$, because then there would have to be hidden variables which lead to a sum of spin squares equal to 2 for all the triads, which is impossible because of the structure of the Kochen-Specker set. Note that $\epsilon$ describes all the imperfections of a real experiment including finite precision but also e.g. imperfect state preparation and non-unit detection efficiency. The value of $N$ and therefore the bound on $\epsilon$ depends on the particular Kochen-Specker set used [2,11].

As noted above, an inevitable requirement for the contradiction to be obtained is the fact that the function $S_{n_{1}}^{2}(\lambda, \mu)$, or in general functions corresponding to at least some switch positions, appear in more than one out of the $N$ triads. This appearance of the same function in different lines of the mathematical proof, corresponding to different experimental contexts, is possible in spite of finite experimental precision only because we defined our observables operationally via the switch positions.

We have shown how non-contextual hidden-variable theories can be disproved by real experiments. This 
shows that Meyer's coloring of a dense subset of the sphere cannot be used to construct non-contextual hidden-variable theories according to the above definition. The values assigned to specific switch positions would have to depend on the context, i.e. on the other switch positions chosen simultaneously by the experimenter. This form of contextuality is a feature of existing explicit models based on Meyer's idea 10. In view of our results, we would assert that the Kochen-Specker theorem is not "nullified" by finite measurement precision. Let us note that arguments in favor of this conclusion were given in 12,13]. Our suggestion how to perform a Kochen-Specker experiment was inspired by some of Mermin's remarks in 12 .

Let us emphasize that using the method of the present paper one can also show that local hidden variables can be disproved in real experiments, e.g. using the GHZ 114 form of Bell's theorem which is also based on sets of propositions that cannot be consistently satisfied by a class of hidden-variable theories (local, in this case). Inequalities corresponding to our bound on $\epsilon$ can be derived and compared to the experimental data [15]. Thus our work confirms the fact that fundamental concepts about the world can indeed be put to experimental test.

When this work was completed, we learned from J.A. Larsson that he has come to similar conclusions using a somewhat related approach 16]. C. S. would like to thank L. Hardy for a useful discussion. This work has been supported by the Austrian Science Foundation (FWF), projects S6504 and F1506, and by the QIPC Program of the European Union.

[1] J. S. Bell, Physics (Long Island City, N.Y.) 1, 195 (1964).

[2] S. Kochen and E. P. Specker, J. Math. and Mech. 17, 59 (1967).

[3] D. Meyer, Phys. Rev. Lett. 83, 3751 (1999).

[4] A. Cabello and G. García-Alcaine, Phys. Rev. Lett. 80, 1797 (1998); C. Simon, M. Żukowski, H. Weinfurter, and A. Zeilinger, Phys. Rev. Lett. 85, 1783 (2000); M. Michler, H. Weinfurter, and M. Żukowski, Phys. Rev. Lett. 84, 5457 (2000).

[5] See e.g. J. F. Clauser and M. A. Horne, Phys. Rev. D 10, 526 (1974). J. F. Clauser and A. Shimony, Rep. Prog. Phys. 41, 1881 (1978).

[6] Meyer's construction was generalized by A. Kent, Phys. Rev. Lett. 83, 3755 (1999).

[7] The principle of experimental tests of classes of hiddenvariable theories is always similar: one verifies that some properties $P_{1}, P_{2}, \ldots P_{n-1}$ hold for almost all systems coming from a certain source by verifying that they hold for sufficiently large random samples. Based on this, one can make a prediction for some property $P_{n}$, which is then again tested on a random sample. This assumes that it is possible for the observer to obtain representative samples. This means that there should be no correlation between the hidden variables of the system under study and the observer's choice which observable to measure. For an experiment where a physical random number generator was used in order to determine the choice of observable to be measured, see G. Weihs, T. Jennewein, C. Simon, H. Weinfurter, and A. Zeilinger, Phys. Rev. Lett. 81, 5039 (1998). It is never possible to exclude "conspiracytype" hidden-variable theories where the decision of the observer which observable to measure is determined by the hidden variables (of the world) in such a way that it is impossible for him to obtain representative samples.

[8] In the present paper we do not discuss stochastic hidden variable theories explicitly. This does not limit the generality of the results derived because the existence of a stochastic local or non-contextual hidden variable model for a given physical system implies that also an underlying deterministic local or non-contextual model can be constructed which reproduces the probabilities of the stochastic model. Therefore e.g. ruling out all possible non-contextual deterministic hidden-variable models implies ruling out all possible non-contextual stochastic models as well. A stochastic hidden-variable theory is non-contextual if for every possible value of the hidden variables the probability to find a certain result for a given observable does not depend on which other observables are measured jointly, and joint probabilities for several observables are simply obtained by multiplication of the probabilities for single observables. Note that because of this last requirement quantum mechanics is not a non-contextual "hidden-variable" theory.

[9] In the ideal case one could define non-contextuality in such a way that the predetermined value of some quantum mechanical observable $X$ is required to be independent of the simultaneously measured observables only if they exactly commute with $X$, i.e. only for ideal Von Neumann measurements. In practice observables corresponding to precise directions do not have an operational meaning. It is evident that this weaker form of non-contextuality cannot be tested experimentally.

[10] Let us note that the models discussed by R. Clifton and A. Kent, quant-ph/9908031, are not non-contextual in the present sense because in these models the result of a measurement of $S_{\vec{n}}^{2}$ in general does not only depend on $\lambda, \mu$, and $\vec{n}$, but also on the other observables measured simultaneously.

[11] A. Peres, J. Phys. A 24, L175 (1991), showed how a contradiction can be demonstrated using 20 triads.

[12] N. D. Mermin, quant-ph/9912081.

[13] D. M. Appleby, quant-ph/000505; D. M. Appleby, quant-ph/0005010; H. Havlicek, G. Krenn, J. Summhammer, and K. Svozil, quant-ph/9911040.

[14] D. M. Greenberger, M. Horne, A. Shimony, and A. Zeilinger, Am. J. Phys. 58, 1131 (1990). For inequalities applicable to the GHZ situation see N. D. Mermin, Phys. Rev. Lett. 65, 1838 (1990).

[15] J.W. Pan, D. Bouwmeester, M. Daniell, H. Weinfurter, and A. Zeilinger, Nature 403, 515 (2000).

[16] J.-A. Larsson, quant-ph/0006134. 